\title{
Introduction to the special issue on the Fourteenth International Conference on Principles and Practice of Constraint Programming (CP 2008)
}

\author{
Peter J. Stuckey
}

Published online: 28 January 2010

(C) Springer Science+Business Media, LLC 2009

This special issue contains five papers that have been presented at the 14th International Conference on Principles and Practice of Constraint Programming (CP 2008) held in Sydney, Australia, September 14-18, 2008, which was held in conjunction with the International Conference on Automated Planning and Scheduling (ICAPS 2008) and the International Conference on Knowledge Representation and Reasoning (KR 2008). Held annually, the CP conference series is the premier international conference on constraint programming.

All 120 submissions to the conference were reviewed by three separate reviewers and extensively discussed. After the final program was selected a subcommittee consisting of myself, Laurent Michel and Michael Trick, considered which papers should be considered for best paper awards. This gave a shortlist of six papers from which the eventual winners were selected. After the conference we invited the authors of these six papers to submit a longer version for the Constraint journal special issue from the conference. The five papers herein were the result.

The first article, "A Hybrid Model for a Multiproduct Pipeline Planning and Scheduling Problem," considers the complexity of managing a large oil pipeline an illustrates the power and flexibility of constraint programming in tackling complex real world problems.

The second article, "A New Framework for Sharp and Efficient Resolution of NCSP with Manifolds of Solutions," modifies interval solving methods to change the axes on which interval propagation is performed to substantially improve the rate of convergence of interval propagation.

The third article, "A Branch and Bound Algorithm for Numerical Max-CSP," defines an innovative approach to Max-CSP for interval problems by simultaneously computing inner and outer approximations of the solutions sets.

P. J. Stuckey $(\bowtie)$

National ICT Australia, Victoria Laboratory, University of Melbourne, Melbourne, Australia e-mail: pjs@csse.unimelb.edu.au 
The fourth article, "CPBPV: A Constraint-Programming Framework For Bounded Program Verification," illustrates how hybrid constraint programming approaches can be applied to program verification to improve upon the current methods used there.

The fifth article, "An MDD-based Generalised Arc Consistency Algorithm for Positive and Negative Table Constraints and Some Global Constraints," shows how multi-decision diagrams can be used as a basis for implementing table constraints and some global constraints in a uniform manner with very fast propagation.

I would like to thank the reviewers, who carefully read and proposed improvements to the five papers published here, and of course the authors, whose hard work to address the recommendations of the reviewers has improved their excellent papers even further. 